C E P R E M A P

CENTRE POUR LA RECHERCHE ECONOMIQUE ET SES APPLICATIONS

Document de travail (Docweb) n ${ }^{\circ} 2005$

\title{
Confinement et chômage en France
}

Malak Kandoussi

François Langot 


\title{
Confinement et chômage ${ }^{1}$
}

Résumé: Nous développons un modèle d'appariement multisectoriel qui reproduit l'impact du premier confinement sur le chômage français, en tenant compte des impacts contrastés entre divers types d'emplois. Nous identifions la taille des restrictions sur les ventes qui ont frappées chaque segment du marché du travail, mais également les recours hétérogènes aux mesures de chômage partiel, mettant ainsi en évidence l'impact inégal du confinement. Ces résultats sont obtenu grâce à un modèle original d'appariement intégrant (i) des risques microéconomiques variants dans le temps, (ii) des externalités de congestion dans le processus de recrutement et (iii) des rigidités de salaires. La persistance générée par notre modèle, nettement plus importante que dans le modèle de Diamond-Mortensen-Pissarides suggère qu'un retour au niveau de chômage d'avant la crise pourrait être atteint en 2023 (2024) dans un scénario à une (double) vague, alors que les mesures de chômage partiel permettent de diviser par deux ces durées d'ajustement.

Mots-clés: COVID-19, chômage, appariement, hétérogénéité des travaileurs

Codes JEL: E24, E32, J64

\section{Quanrantine and unemployment}

\begin{abstract}
We develop a multisectoral matching model that reproduces the impact of the first confinement on French unemployment, taking into account the contrasting impacts between different types of jobs. We identify the size of the sales restrictions that hit each segment of the labour market, but also the heterogeneous use of short-time working measures, thus highlighting the uneven impact of the confinement. These results are obtained using an original matching model integrating (i) timevarying microeconomic risks, (ii) congestion externalities in the recruitment process, and (iii) wage rigidities. The persistence generated by our model, which is much greater than in the DiamondMortensen-Pissarides model, suggests that a return to the pre-crisis level of unemployment could be achieved in 2023 (2024) in a single (double) wave scenario, whereas short-time working measures make it possible to halve these adjustment durations.
\end{abstract}

Keywords:COVID-19, unemployment, matching, worker heterogeineity

JEL Codes: E24, E32, J64

${ }^{1}$ F. Langot a bénéficié du soutien financié de l'Institut Universitaire de France, des progammes de recherche PANORisk et de EUR grant ANR-17EURE-0001. Merci à T.Brand et D. Cohen pour leurs remarques.

${ }^{2}$ Contact: malak.kandoussi@univ-evry.fr. Université Paris-Saclay, Université d’Evry, EPEE, 91025, Evry-Courcouronnes, France

${ }^{3}$ Contact : flangot@univ-lemans.fr. Université du Mans (Gains-TEPP IRA), IUF, EEP, Cepremap, IZA 


\section{Introduction}

La récente crise de la COVID-19 a eu un impact sans précédent sur le marché du travail français, tant par son amplitude que par sa rapidité : le taux de chômage est passé de $7,7 \%$ en février 2020 à $27,5 \%$ en avril 2020, si on inclut les personnes en chômage partiel. I Après ce pic atteint en deux mois, l'amplitude et la rapidité de la baisse du chômage à également été spectaculaire : en septembre, il "n'y avait plus que" 9,7\% de chômeurs, incluant ceux en chômage partiel. Ces ajustements sont très différents de ceux observés dans les crises précédentes : Hall and Kudlyak (2020) soulignent que depuis 70 ans, la réduction annuelle du taux de chômage est de 0,55 point de pourcentage par an après tous les pics des différentes récessions observées aux États Unis. En France, il a fallu attendre des années avant de voir "la courbe du chômage s'inverser" après la crise de 2008.2

La crise de la COVID-19 est donc différente des précédentes : elle nécessite une analyse particulière. Si les précédentes crises ont incité à trouver des mécanismes théoriques générant suffisamment de persistance après un pic de chômage, cette dernière crise nous conduit à mettre en avant des mécanismes expliquant (i) une très forte montée du chômage et (ii) sa résorption rapide. La sévérité du confinement et son accompagnement par le chômage partiel jouent tous les deux un rôle majeur. Il existe des restrictions sur les échanges qui empêchent les entreprises de produire mais aussi de vendre à des consommateurs moins nombreux. Face à cet effondrement des échanges, les entreprises peuvent se séparer de certains de leurs employés, sans pour autant rompre leurs contrats, ce qui permettra une reprise du travail plus rapide. Dans cet article, nous proposons une modélisation parcimonieuse des ajustements du marché du travail français pendant la crise de la COVID-19 dans le cadre d'un modèle à la Diamond (1982)-Mortensen (1982)-Pissarides (1985) (DMP). [ $^{3}$ La modélisation structurelle permet aussi d'évaluer des scénarios différents à celui effectivement observé, comme par exemple un confinement plus souple mais plus long.

Notre premier défi avec la crise du COVID-19 est d'identifier l'ampleur et la persistance des chocs qui frappent chaque type d'activité économique, sachant que les premières enquêtes empiriques de Adams-Prassl et al. (2020) $)^{4}$ Fana et al. (2020) ${ }^{5}$ ou encore Givord

1. Afin de comptabiliser ces personnes, nous utilisons la statistiques du nombre de chômeur partiel en Équivalent Temps Plein (ETP). Voir DARES (2020)

2. Voir Guggemos and Vidalenc (2014) pour plus de détails

3. La recherche d'une solution parcimonieuse est importante pour la modélisation macroéconomique, étant donnée que cet épisode restera à jamais dans les séries statistiques et devra donc être pris en compte lors des futures analyses macroéconomiques.

4. Adams-Prassl et al. (2020) montrent que, pour certaines professions, la capacité des travailleurs à effectuer un grand pourcentage de leurs tâches à domicile (c'est-à-dire le " télétravail ") est limitée ; cela est particulièrement vrai pour les professions à faible revenu.

5. En utilisant l'enquête de l'Union européenne sur les forces de travail, Fana et al. (2020) montrent que la plupart des effets négatifs des mesures de verrouillage sont concentrées sur les travailleurs moins qualifiés. En effet, les secteurs fermés de force par décret de leur décomposition se caractérisent par des salaires bas et des taux de cessation d'emploi élevés. 
and Silhol (2020) et Jauneau and Vidalenc (2020) pour la France ${ }^{6}$ ont déjà souligné les effets hétérogènes du confinement sur le marché du travail. Pour contrebalancer les restrictions du confinement, le gouvernement français a mis en place des mesures de chômage partiel. Bien entendu, ces mesures permettent de garantir un revenu aux consommateursoffreurs de travail, mais, au-delà, elles permettent aussi un retour plus rapide et moins coûteux au travail lors des phases de dé-confinement. Il est donc central d'identifier cette chronique de chocs, alternant restrictions puis accompagnements à la reprise de l'emploi. Nous traitons ici le confinement et le dé-confinement comme des "chocs" car ce sont des mesures sanitaires ayant un objectif de santé publique ne pouvant donc pas être déduite d'un modèle visant uniquement à décrire des arbitrages économiques.

Le deuxième défi consiste à étendre le modèle DMP de manière à pouvoir expliquer les grandes crises. Cela représente un défi pour ce modèle pour les quatre raisons suivantes: (i) le taux de chômage a plus que doublé en un mois, soulignant la très forte élasticité du chômage par rapport à la conjoncture; (ii) dans le passé, à la suite d'une crise, le taux de chômage n'a jamais connu de baisse rapide, ce qui suggère que les externalités d'appariement ne sont pas suffisantes; ( iii) l'augmentation du risque de chômage pendant la crise dépend fortement du type considéré de travailleur; 7 (iv) la distribution de la productivité au sein de chaque profession varie au cours du cycle, avec une hétérogénéité croissante pendant les périodes de récession. 8

Une calibration de cette extension du modèle DMP, visant à reproduire la dynamique mensuelle des indicateurs du marché du travail français depuis mars 2020, permet de révéler l'ampleur des "chocs COVID-19", spécifiques à chaque type d'activité. Les chocs représentant les restrictions induites par le confinement sont estimés et peuvent être interprétés comme les contraintes effectives qui ont frappées de manière inattendue et différenciée chaque type d'activité. D'un autre côté, les chocs sur les coûts associés à la reprise d'activité d'un poste de travail pendant la période de déconfinement peuvent aussi être identifiés et interprétés comme l'efficacité productive des mesures de chômage partiel. La baisse quantitative de la production atteint $30 \%$ en avril sur les postes employant des non-diplômés, soit une perte de valeur créée par poste de $26 \%$, alors que sur les postes employant des individus ayant un diplôme supérieur à $\mathrm{Bac}+2$, la perte en volume est quasi-nulle pour une perte de valeur créée par poste de $6 \%$. Le corollaire en terme de chômage (incluant le chômage partiel) est une hausse de 32 points de pourcentage (pp)

6. En utilisant L'enquête Épidémiologie et conditions de vie (EpiCov) mise en place par la Drees, l'Inserm, Santé Publique France et l'Insee, Givord and Silhol (2020) montrent que 80\% des cadres en activité déclarent télé travailler la semaine avant l'enquête tandis que seulement $6 \%$ des ouvriers ont eu recours au télétravail. De plus Jauneau and Vidalenc (2020) expliquent que selon l'enquête Emploi 2019 et 2020 de l'Insee, la durée de temps de travail a moins reculé pour les professions ayant pu recourir au télétravail (majoritairement des cadres) tandis que le volume d'heures a largement diminué pour les professions dont la possibilité de télé-travailler étaient limitées ou dont l'activité s'est totalement arrêtée avec le confinement.

7. Robin (2011) et Lise and Robin (2017) montrent que l'hétérogénéité est importante pour rendre compte de la dynamique globale du marché du travail. Voir aussi Ferraro (2018) \& (2000) et Adjemian et al. (2019).

8. Cette régularité empirique a été soulignée par Bloom (2009). 
et autour de 10 pp pour ceux ayant au moins un $\mathrm{Bac}+2$. Comme les embauches étaient nulles en avril, ces hausses du chômage sont entièrement déterminées par les fermetures de postes de travail. Pour rendre compte de la rapidité de la résorption de ces niveaux de chômage lors du déconfinement, nous évaluons que le chômage partiel revient à baisser de $90 \%$ les coûts unitaires de reprise de l'activité d'un poste employant un non-diplômé et de $7 \%$ ceux associés à la reprise d'activité des personnes ayant au moins un $\mathrm{Bac}+2$. Un scénario sans ces aides à la reprise d'emploi liées au chômage partiel, et sans deuxième vague, prévoit que le taux de chômage agrégé aurait été de 3 pp plus élevé en janvier 2021 qu'il ne l'était en février 2020 (soit un taux de chômage à 10,7\%), alors qu'il ne sera "que" de 2 pp plus élevé grâce au chômage partiel (soit un taux de chômage à 9,7\%). Évidement, les mesures de chômage partiel facilite et donc amplifie les mises à pied : comme l'employeur anticipe que la reprise d'emploi sera moins coûteuse, il aura tendance à se séparer plus facilement de ses employés, la stratégie dite de "rétention de la main d'oeuvre" étant moins valorisée. Mais ces forts ajustements à la hausse du chômage sont compensés par la très forte reprise d'activité dès le début du déconfinement. Nous montrons qu'une stratégie de confinement moins stricte mais plus longue permet de revenir plus vite au niveau de chômage d'avant crise, de par son effet plus modéré sur les séparations, un confinement moins stricte permettant de laisser ouvert davantage de postes. Finalement un scénario a deux vagues (mars puis octobre), laisse prévoir un retour du chômage à son niveau d'avant la crise en janvier 2023.

Il existe déjà une littérature importante étudiant l'impact de la crise du COVID-19 sur le marché du travail. De nombreuses études empiriques, dont Gallant et al. (2020), Barrero et al. (2020), Bartik et al. (2020) entre autres, attestent de l'ampleur sans précédent de la baisse des embauches et de l'augmentation des départs, toutes deux conduisant à la flambée du chômage aux États-Unis. Sur les données françaises, L'enquête Épidémiologie et conditions de vie (EpiCov) mise en place par la Drees,l'Inserm, Santé Publique France et l'Insee dans le contexte de la pandémie de Covid-19 montre qu'en mai 2020, 26, 9\% des personnes étant en emploi avant le confinement déclarent ne pas avoir travaillé la semaine précédant l'enquête. 9 Des travaux basés sur des approches structurelles améliorent notre compréhension des mécanismes de propagation de cette crise, comme ceux de Gregory et al. (2020), Hall and Kudlyak (2020) ou Bernstein et al. (2020).10 Notre article complète ces précédentes études en déterminant le modèle structurel le plus parcimonieux permettant de rendre compte quantitativement de l'impact de la crise du COVID-19 sur le marché du travail français, intégrant donc les particularités liées au chômage partiel.

9. voir Givord and Silhol (2020)

10. Notons également que Kapicka and Rupert (2020) et Birinci et al. (2020) intègrent des modèles de type DMP dans des modèles épidémiologiques afin de mieux analyser l'interaction entre les politiques de santé publique et l'efficacité économique. Cette approche normative très intéressante sort du cadre du présent article. 


\section{Modèle}

Nous analysons l'effet des mesures de confinement (non-anticipées) avec un modèle d'appariement. Nous ajoutons au modèle de DMP deux externalités importantes qui visent toutes deux à rendre compte des plus grandes difficultés en temps de crise. Ainsi, on suppose que les coûts unitaires de recrutement augmenteront lorsque le chômage s'écarte de sa valeur à long terme (afflux de CV par annonce...), ce qui introduit une externalité de congestion sur les embauches. Nous supposons également que la dispersion des productivités idiosyncratiques est plus grande lorsque le chômage diverge de sa valeur à long terme, ce qui rend contra-cyclique l'incertitude microéconomique.

Nous supposons également que les marchés du travail sont segmentés, chaque compétence n'accomplissant qu'une seule tâche. Les interactions entre ces marchés résultent des choix de consommation des ménages, ce qui permet de déterminer les prix relatifs à chaque période et donc d'avoir des interactions d'équilibre entre les différents marché du travail.

\subsection{Consommateurs-offreurs de travail}

Les consommateurs-offreurs de travail sont neutres au risque et se caractérisent par leur compétence $j \in \mathcal{J}$. Les préférences de chaque travailleur $i$ sont définies sur un ensemble de biens $s \in \mathcal{S}$ de taille $S$. Les préférences de chaque agent $i$ avec la compétence $j$ sont définies comme suit :

$$
C_{i, j, t}^{L}=S^{\frac{1}{1-\sigma}}\left(\sum_{s \in \mathcal{S}}\left(C_{i, j, s, t}^{L}\right)^{\frac{\sigma-1}{\sigma}}\right)^{\frac{\sigma}{\sigma-1}}
$$

La contrainte de ressources est

$$
I_{i, j, t}=\sum_{s \in \mathcal{S}} p_{s, t} C_{i, j, s, t}^{L}=p_{t} C_{i, j, t}^{L} \quad \text { pour } \quad I_{i, j, t}=\left\{w_{i, j, t}(\alpha), b_{i, j}\right\} \quad \forall j \in \mathcal{J},
$$

où l'indice des prix à la consommation (IPC) est $p_{t}=\left(\frac{1}{S} \sum_{s \in \mathcal{S}} p_{s, t}^{1-\sigma}\right)^{\frac{1}{1-\sigma}}$, et $w_{i, t}(\alpha)$ est le salaire réel et $b_{i}$ l'allocation chômage. La demande optimale pour chaque bien $s \in \mathcal{S}$ est

$$
C_{i, j, s, t}^{L}=\left(\frac{p_{s, t}}{p_{t}}\right)^{-\sigma} \frac{C_{i, j, t}^{L}}{S}
$$

Les fonctions valeur de chaque travailleur sont

$$
\begin{aligned}
W_{i, j, t}(\alpha) & =w_{i, j, t}(\alpha)+\beta\left[\left(1-s_{j, t+1}\right) \int_{\alpha_{j, t+1}^{r}}^{\infty} W_{i, j, t} \frac{d G(\alpha)}{1-G\left(\alpha_{j, t+1}^{r}\right)}+s_{j, t+1} U_{i, j, t+1}\right] \\
U_{i, j, t} & =b_{i, j}+\beta\left[f_{j, t+1}\left(1-s_{j, t+1}\right) \int_{\alpha_{j, t+1}^{r}}^{\infty} W_{i, j, t} \frac{d G(\alpha)}{1-G\left(\alpha_{j, t+1}^{r}\right)}+\left(1-f_{j, t+1}\left(1-s_{j, t+1}\right)\right) U_{i, j, t+1}\right],
\end{aligned}
$$

où $\beta$ est le facteur d'actualisation, $s_{j, t}$ le taux endogène de cessation d'emploi et $f_{j, t}$ le taux de rencontre entre un demandeur d'emploi au chômage et un poste vacant. 


\subsection{Flux sur le marché du travail}

La population des offreurs de travail est constituée de $S$ type de qualification, dont les masses dans la population active sont fixées et notées $\omega_{s}$, avec $\sum_{s=1}^{S} \omega_{s}=1$.

Une fonction d'appariement génère les rencontres entre emplois vacant et chômeurs, alors que les séparations résultent d'un processus de sélection des travailleurs les plus productifs au sein de chaque entreprise. Le marché du travail est segmenté par compétence $j \in \mathcal{J}$, et chaque compétence $j$ ne peut produire qu'un seul type de bien $s$. Au début de chaque période $t$, le nombre de travailleurs au sein de l'entreprise est la somme des embauches de la période précédente $\left(q_{s, t-1} V_{s, t-1}\right)$ et du précédent stock d'emplois $\left(N_{s, t-1}\right)$. Ensuite, dans chaque entreprise $i$ du secteur $s$, un choc idiosyncratique se révèle et la productivité du travailleur $\left(\alpha_{i, s, t}\right)$ devient observable. Il y a séparation si $\alpha<\alpha_{i, s, t}^{r}$. Ce seuil détermine la masse des séparations endogènes. Ainsi les séparations de la période incluent les anciennes et les nouvelles embauches. Le choc microéconomique $\alpha$ est tiré dans la distribution $G_{s, t}(\alpha)$, qui est une distribution log-normale avec une moyenne $\mu_{G}$ et une variation $\sigma_{s, t}$. Cette distribution est variable dans le temps. Pour tenir compte de l'augmentation du risque microéconomique en période de récession, nous supposons que

$$
\sigma_{t}=\sigma_{G}\left(\frac{U_{t}}{U}\right)^{\xi}
$$

où le niveau actuel du taux de chômage $U_{t}$ et sa valeur à long terme $U$ sont pris comme donnés au niveau de l'entreprise $i$ sur le segment du marché du travail $s$. Le paramètre $\xi$ contrôle l'impact de la récession sur $\sigma_{t}$. П⿴囗十 Une fois que l'information sur la productivité est révélée, le stock d'emplois disponibles pour la production peut être déterminé. La négociation salariale peut alors avoir lieu et, finalement la production. Ce n'est qu'à la fin de la période $t$ que les stocks de chômage $\left(U_{s, t}\right)$ et d'emploi $\left(N_{s, t}\right)$ sont définis, permettant de déterminer de nouveaux appariements qui se produisent gràce au nombre d'emplois vacants $V_{s, t}$, qui seront pourvus chacun avec une probabilité $q_{s, t}$.

Les travailleurs et les entreprises dirigent leurs efforts de recherche vers le sous-marché correspondant, puis vers un secteur, avec $j=s$. Suivant Den Haan et al. (2000), la fonction d'appariement de chaque segment du marché du travail est

$$
M_{s}\left(U_{s, t}, V_{s, t}\right)=\frac{U_{s, t} V_{s, t}}{\left(U_{s, t}^{\tau_{s}}+V_{s, t}^{\tau_{s}}\right)^{1 / \tau_{s}}},
$$

Les probabilités qu'un chômeur trouve un emploi $f_{s}\left(\theta_{s, t}\right)=\frac{M\left(U_{t}, V_{s, t}\right)}{U_{s, t}}=\left(1+\theta_{s, t}^{-\tau_{s}}\right)^{-1 / \tau_{s}}$ et que celle qu'un emploi vacant soit pourvu $q_{s}\left(\theta_{s, t}\right)=\frac{M\left(U_{s, t}, V_{s, t}\right)}{V_{s, t}}=\left(1+\theta_{s, t}^{\tau_{s}}\right)^{-1 / \tau_{s}}$ sont toutes deux dans l'intervalle $[0 ; 1]$. La dynamique de l'emploi est alors donnée par

$$
N_{s, t}=\left(1-s_{s}\right)\left(1-G_{s, t}\left(\alpha_{s, t}^{r}\right)\right)\left(N_{s, t-1}+q\left(\theta_{s, t-1}\right) V_{s, t-1}\right) \text {, }
$$

11. La contra-cyclicité du risque microéconomique au niveau de l'entreprise est documentée par Bloom (2009) et Bloom et al. (2018). 
où $0<s_{s}<1$ est la probabilité exogène de destruction d'emplois. Le taux de séparation est défini par $J S R_{t} \equiv s_{s, t}=s_{s}+\left(1-s_{s}\right) G_{s, t}\left(\alpha_{s, t}^{r}\right)$, et il donne les entrées au chômage, compte tenu des informations de la période $t$. Le taux de reprise d'emploi est défini par $J F R_{t} \equiv f_{s, t}=\left(1-s_{s, t+1}\right) f_{s}\left(\theta_{s, t}\right)$, et il donne les sorties du chômage, en tenant compte non seulement des informations de la période $t$ mais aussi de celles de la période $t+1$. Enfin, la normalisation de la taille de la population à l'unité conduit à $\sum_{s=1}^{S} \omega_{s}\left(U_{s, t}+N_{s, t}\right)=1$.

\subsection{Les entreprises}

Pour l'entreprise $i$ du secteur $s$, les embauches résultent d'un processus de recherche qui consiste à afficher des postes vacants $V_{i, s, t}$ qui seront pourvus par des chômeurs avec une probabilité $q_{s, t}$. Le coût unitaire, en unités de production, de chaque poste vacant est

$$
\kappa_{i, s, t}=\kappa_{s, t}=\kappa_{s}\left(\frac{U_{s, t}}{U_{s}}\right)^{\gamma_{s}} \forall i
$$

où le niveau du taux de chômage $U_{s, t}$ et sa valeur de long terme $U_{s}$ sont pris comme donnés au niveau de l'entreprise, ce qui conduit à interpréter la composante variable dans le temps du coût d'un emploi vacant comme une externalité de congestion. 12 Étant donné que $\gamma_{s}$ dépend de $s$, cette externalité de congestion est spécifique au secteur. Les coûts unitaires sont plus élevés en période de récession, car chaque emploi vacant (qui est rare en récession) reçoit un très grand nombre de candidatures (le nombre de chômeurs étant important en récession). Par conséquent, les récessions augmentent le coût du traitement pour chaque candidature. ${ }^{13}$ Hall and Kudlyak (2020) montrent que cet effet de congestion est important pour reproduire la persistance observée du chômage après une récession.

Soit $\widetilde{\alpha}_{s, t}=\frac{\int_{\alpha_{s, t}^{r}}^{+\infty} \alpha d G_{s, t}(\alpha)}{1-G_{s, t}\left(\alpha_{s, t}^{r}\right)}$ la productivité moyenne des emplois d'une entreprise fabriquant des biens $s$, alors la fonction de production est ${ }^{14} Y_{s, t}=A_{s, t} \widetilde{\alpha}_{s, t} N_{s, t}$, où $A_{s, t}$ est la Productivité Globale des Facteurs (PGF) spécifique à la qualification $s$. Soit $\widetilde{w}_{s, t}=$ $\frac{\int_{\alpha_{s, t}^{r}}^{+\infty} w_{s, t}(\alpha) d G_{s, t}(\alpha)}{1-G_{s, t}\left(\alpha_{s, t}^{r}\right)}$ le salaire moyen, l'objectif de l'entreprise est de maximiser ses bénéfices actualisés :

$$
\max \sum_{\tau=0}^{+\infty} \beta_{t+\tau}^{\tau} D_{s, t+\tau}=\max \sum_{\tau=0}^{+\infty} \beta_{t+\tau}^{\tau}\left\{p_{s, t+\tau} Y_{s, t+\tau}-\widetilde{w}_{s, t+\tau} N_{s, t+\tau}-\kappa_{s, t+\tau} V_{s, t+\tau}\right\}
$$

12. Nous choisissons la même forme fonctionnelle que Hall and Kudlyak (2020), mais nous introduisons un paramètre spécifique au secteur $\gamma_{s}$ qui induit un externalité de congestion spécifique au secteur.

13. Blanchard and Diamond (1994) ont été les premiers à donner des fondements de ces coûts unitaires d'embauche contra-cycliques, basés sur l'existence d'externalités d'échange : ils montrent que dans un marché du travail où les entrepreneurs préfèrent embaucher des chômeurs de courte durée, les récessions conduisent à une augmentation de la part des chômeurs de longue durée qui encombrent alors le processus d'embauche. Engbom (2019) et Molavi (2018) suggèrent que les coûts unitaires d'embauche contra-cycliques sont en adéquation avec les données.

14. Dans ce qui suit, nous omettons pour simplifier l'indice $i$, qui désigne la firme $i$ dans chaque secteur $s$ car l'équilibre est symétrique à l'intérieur des secteurs. 
sous la contrainte (1) et les conditions d'exclusion de Kuhn et Tucker, données par 15

$$
q_{s}\left(\theta_{s, t}\right) V_{s, t} \geq 0, \quad \lambda_{s, t} \geq 0, \quad \text { et } \quad \lambda_{s, t} q_{s}\left(\theta_{s, t}\right) V_{s, t}=0 .
$$

\subsection{Salaires}

Le salaire d'équilibre est déterminé par une règle de partage à la Nash entre un travailleur et un employeur où $\eta_{s} \in(0,1)$ est le pouvoir de négociation du travailleur et $b_{s}$ est le coût d'opportunité de l'emploi. Ces paramètres sont spécifiques à chacune des qualifications. De plus, comme cela est maintenant usuel dans les applications quantitatives du modèle DMP, une rigidité salariale est introduire ${ }^{16}$ Les rigidités de salaire réel ont été remise sous les projecteurs par Cortes and Forsythe (2020) lors de la crise de la COVID-19. ${ }^{17}$ Il existe plusieurs manières d'introduire des rigidités salariales dans un modèle DMP : (i) un jeu de négociation d'offre alternée comme dans Hall and Milgrom (2008) ou (ii) l'introduction d'une norme salariale ou consensus social comme dans Hall (2005). Suivant Blanchard and Gali (2010) ou Leduc and Liu (2019), nous adoptons la deuxième stratégie de modélisation, sachant que ses implications sont assez similaires à la première. Par conséquent, le salaire réel est une moyenne pondérée entre le salaire négocié à la Nash et le salaire de l'état stationnaire considéré comme la norme :

$$
w_{s, t}(\alpha)=\varrho_{s}\left[\eta_{s}\left(p_{s, t} \alpha A_{s, t}+p_{s, t} \kappa_{s, t} \theta_{s, t}\right)+\left(1-\eta_{s}\right) b_{s}\right]+\left(1-\varrho_{s}\right) w_{s}
$$

où le paramètre $\varrho_{s} \in[0,1]$ mesure la rigidité salariale spécifique à la compétence $s$, et $w_{s}$ le salaire moyen de l'état stationnaire des travailleurs de type $s$. Suivant Daly et al. (2012) 18, nous supposons que la rigidité de salaire diminue avec le niveau d'éducation. Par conséquent, nous supposons que $\varrho_{s}=a_{\varrho} x+b_{\varrho}$ où $a_{\varrho}<0$.

\subsection{Equilibre}

Dans ce qui suit, nous normalisons l'IPC à l'unité : $p_{t}=1, \forall t$. Afin d'avoir un équilibre tenant compte de l'ensemble des rémunérations, il est nécessaire d'introduire des "capitalistes". Ils constitueront $(1-\varphi) \%$ de la population totale. Leurs revenus à chaque date $t$ sont les profits des entreprises $\left(\sum_{s} D_{s, t}\right)$. On suppose que ces individus sont neutres face

15. Voir l'annexe A pour plus de détails sur la solution du problème de l'entreprise.

16. Voir, entre autres, Blanchard and Gali (2010), Christiano et al. (2016), Leduc and Liu (2019) ou encore Nicolas Petrosky and Zhang (2020). Ces articles montrent que les modèles DSGE avec un marché du travail à la DMP doivent introduire des rigidités de salaire réel pour rendre compte des caractéristiques observées du cycle économique. Les études empiriques de Kurmann and McEntarfer (2019) et Jardim et al. (2019) montrent qu'environ $20 \%$ des personnes ayant conservé leur emploi ont subi des réductions de salaire nominales pendant la récession, tandis que moins de $10 \%$ ont vu leurs gains gelés. Voir aussi l'enquête de Elsby and Solon (2019).

17. Ils montrent que les revenus des travailleurs qui restent employés pendant la crise de la COVID-19 ne changent pas de façon atypique pendant cette période.

18. Voir https://www.frbsf .org/economic-research/indicators-data/nominal-wage-rigidity/ pour les données mises à jour jusqu'en 2020 
au risque et ont les mêmes préférences que les consommateurs-offreurs de travail : leur panier de biens de consommation est $C_{t}^{K} \cdot 19$

Demande. La demande agrégée pour chaque secteur $\left(Y_{s, t}^{D}\right)$ est donnée par

$$
Y_{s, t}^{D}=p_{s, t}^{-\sigma}\left(\frac{\varphi \sum_{j \in \mathcal{S}} \omega_{j} C_{j, t}^{L}+(1-\varphi) C_{t}^{K}}{S}\right),
$$

impliquant que la demande globale est $Y_{t}^{D}=\sum_{s \in \mathcal{S}_{t}} p_{s, t} Y_{s, t}^{D}$.

Offre. Sur chaque marché, l'offre globale $Y_{s, t}^{S}$ est donnée par

$$
Y_{s, t}^{S}=Y_{s, t}-\kappa_{s, t} V_{s, t}
$$

impliquant que l'offre agrégée est $Y_{t}^{S}=\sum_{s \in \mathcal{S}} p_{s, t} \omega_{s} Y_{s, t}^{S}$.

Prix d'équilibre. Étant donné que $Y_{s, t}^{D}=Y_{s, t}^{S} \equiv Y_{s, t}^{*}$ à l'équilibre, $\forall s$ - ce qui implique $Y_{t}^{D}=Y_{t}^{S} \equiv Y_{t}^{*}$ - les prix d'équilibre sont déduits de

$$
p_{s, t}=\left(\frac{1}{S} \frac{Y_{t}^{*}}{Y_{s, t}^{*}}\right)^{\frac{1}{\sigma}} \quad \forall s \in \mathcal{S} .
$$

Marché du travail. En utilisant l'équation de salaire (équation (2)), nous obtenons la condition de destruction d'emplois (productivité de réservation), et la condition de création d'emplois (embauches) :20

$$
\begin{aligned}
\alpha_{s, t}^{r} & =\max \left\{0 ; \frac{1}{\left(1-\eta_{s}\right) p_{s, t} A_{s, t}}\left(\left(1-\eta_{s}\right) b_{s}+\eta p_{s, t} \kappa_{s, t} \theta_{s, t}-\frac{p_{s, t} \kappa_{s, t}}{q\left(\theta_{s, t}\right)}-\lambda_{s, t}\right)\right\} \\
\frac{p_{s, t} \kappa_{s, t}}{q_{s}\left(\theta_{s, t}\right)}-\lambda_{s, t} & =\beta\left[\left(1-s_{s, t+1}\right)\left(p_{s, t+1} A_{s, t+1} \widetilde{\alpha}_{s, t+1}-\widetilde{w}_{s, t+1}+\frac{p_{s, t+1} \kappa_{s, t+1}}{q_{s}\left(\theta_{s, t+1}\right)}-\lambda_{s, t+1}\right)\right]
\end{aligned}
$$

Crise de la COVID-19 : restrictions liées au confinement et chômage partiel. La crise de la COVID-19 est modélisée comme un choc d'offre venant restreindre transitoirement les possibilités physiques de vente des entreprises. Ceci peut donc être assimilé à un choc négatif de $\mathrm{PGF}, A_{s, t}$. Chaque secteur est affecté de façon spécifique, ce qui reflète, par exemple, des possibilités hétérogènes de télé-travail.

Ce choc affecte le revenu des ménages via les ajustements de salaire et d'emploi : la demande adressée aux entreprises est donc modifiée, ainsi que sa composition entre les $S$ biens, ce qui va modifier les prix relatifs $p_{s, t}$. In fine, les restrictions induites par le

19. La valeur de $\varphi$ n'a pas d'incidence sur l'équilibre. Dans la partie numérique, nous prendrons une valeur de $\varphi$ égale à $2 \%$ (voir Kandoussi and Langot (2020)).

20. Voir l'annexe A pour plus de détails. 
confinement auront donc un impact sur la valeur marginale de chaque bien produit via les variations de $p_{s, t} A_{s, t}$.

Le chômage partiel permet aux entreprises de ne pas payer les coûts d'une main d'oeuvre qui ne produit plus sous la contrainte du confinement : en ce sens, il s'apparente au chômage où le travailleur n'est plus dans les comptes de l'entreprise, que cela soit dans la VA ou dans la masse salariale, mais perçoit malgré tout une rémunération, sous forme d'une indemnité. C'est lors de la phase de reprise d'activité que le chômage partiel se distingue du chômage traditionnel : la reprise du travail des chômeurs partiels, restés sous contrat, est moins coûteuses, même si elle reste onéreuse du fait des différentes procédures qu'il est nécessaire de gérer pour l'entreprise. Cela se traduit dans le modèle par une baisse transitoire des coûts unitaires de vacances d'emploi $\kappa_{s, t}$, reflétant la plus grande facilité de la reprise d'activité d'un chômeur partiel. Comme le coût $\kappa_{s, t}$ est croissant avec le chômage spécifique à la qualification, la baisse du coût liée au chômage partiel sera donc proportionnelle au nombre de chômeurs partiels sur chaque segment du marché du travail.

\section{Résultats quantitatifs}

\subsection{Calibration}

Le modèle est mensuel. Chaque "secteur" représente la production de travailleurs identifiés par leurs niveaux de formation. ${ }^{21}$

Calibrations basées sur des informations extérieures. Basée sur un taux d'actualisation moyen des données internationales de $5,37 \%$ par an, nous fixons $\beta=1 /(1+$ $0.0573)^{1 / 12} \approx 0.9953$. Nous supposons que les distributions des $\alpha$ sur chacun des segments $s$ est log-normale. Nous les restreignons à être identique pour chaque sous-population, avec une moyenne nulle et un écart type égal à 0.12, comme dans Krause and Lubik (2007). Comme dans Den Haan et al. (2000) ou Krause and Lubik (2007), on impose que $68 \%$ des séparations soient exogènes à l'état stationnaire, et ce sur tous les segments du marché du travail. Les paramètres $\left\{\sigma, \xi,\left\{\tau_{s}\right\}_{s=1}^{S},\left\{\gamma_{s}\right\}_{s=1}^{S}, a_{\varrho}, b_{\varrho}\right\}$ sont repris de Kandoussi and Langot (2020) qui estiment ce modèle sur la crise de 2008 aux Etats-Unis. L'ensemble de ces paramètres sont dans le tableau 1

Calibrations basées sur les restrictions de l'état stationnaire et des données pré-COVID-19. Les données utilsées se réfèrent à l'année 2016, soit un an après la sortie de la crise financière. La part de la population $\left(\omega_{s}\right)$ ainsi que le taux de chômage spécifique à chaque qualifiaction $\left(U_{s}\right)$ sont construits à partir de Beck and Vidalenc (2017) Les taux d'embauche par diplôme $\left(J F R_{s}\right)$ viennent de Fontaine et al. (2020). A l'état stationnaire, ces moments sont liées par la restriction suivante $U R_{s}=\frac{J S R_{s}}{J S R_{s}+J F R_{s}}$, ce qui

21. Comme cette caractéristique ne change pas après l'entrée sur le marché du travail, cette segmentation justifie notre hypothèse d'absence de mobilité entre les secteurs/segments du marcheé du travail. 


\begin{tabular}{cccccc}
\hline \hline Paramètres & $\beta$ & $\sigma$ & $\xi$ & $a_{\varrho}$ & $b_{\varrho}$ \\
Communs & 0.9953 & 4 & 0.5 & -0.17 & 0.67 \\
\hline \hline & & LHS & HS & Coll. & Bach. \\
\hline Paramètres & $\tau_{s}$ & 1.4 & 1.5 & 1.5 & 1.6 \\
spécifiques & $\gamma_{s}$ & 0.8 & 0.6 & 0.5 & 0.37 \\
\hline \hline
\end{tabular}

\section{TABLE 1 - Paramètres fixés sur la base d'informations extérieures}

nous donne les valeurs cohérentes des taux de séparation $\left(J S R_{s}\right)$. Comme les données de Fontaine et al. (2020) se décomposent qu'en trois niveau de diplôme, agrégeant tous ceux ayant plus que la bac, nous supposons que le taux d'embauche de ceux ayant un diplôme universitaire est le même que ceux ayant un $\mathrm{Bac}+2$. Les taux de séparation par niveau de

\begin{tabular}{c|cccc|c}
\hline \hline & non dipl & CAP-BEP & Bac-Bac+2 & Sup & \\
\hline$J F R$ & 0.16 & 0.2 & 0.25 & 0.25 & 0.22 \\
$J S R$ & 0.035 & 0.024 & 0.023 & 0.015 & 0.0235 \\
$U R$ & 0.179 & 0.108 & 0.0842 & 0.057 & 0.1003 \\
Part de la population & $17 \%$ & $25 \%$ & $36 \%$ & $22 \%$ & $100 \%$ \\
\hline \hline
\end{tabular}

\section{TABLE 2 - Les flux et le stock des travailleurs (2016)}

diplôme $\left(J S R_{s}\right)$ donnent les valeurs d'équilibre de $\alpha_{s}^{r}$, et les taux d'embauche par diplôme $\left(J F R_{s}\right)$ donnent les valeurs d'équilibre de $\theta_{s}$. En appliquant les définitions de $U R_{s}$, nous pouvons alors déduire $V_{s}$ à l'état stationnaire. Enfin, avec la distribution log-normale de $\alpha$, nous en déduisons $\widetilde{\alpha}_{s}$. Le tableau 2 résume l'ensemble de ces moments. En utilisant les équations ((3) et (4) ) prises à l'état stationnaire et en supposant que $\kappa_{s}$ est proportionnel à $A_{s}$, ie. $\kappa_{s}=k A_{s}$, on identifie $\eta_{s}$ et $\widetilde{b}_{s} \equiv b_{s} /\left(p_{s} A_{s}\right)$, qui sont donc spécifiques au niveau de diplôme. La valeur de $k$ est choisie de telle sorte que le pouvoir de négociation moyen $\left(\eta=\sum_{s} \omega_{s} \eta_{s}\right)$ soit égal à 0.5 : on obtient $k=0.1184$. Les $\left\{A_{s}\right\}_{s=1}^{S}$ sont déterminées en minimisant la distance entre le salaires relatifs spécifiques à chaque qualification du modèle et ceux des données. 22 Tous les résultats obtenus via ces restrictions d'état stationnaire sont dans le tableau 3

\begin{tabular}{cccccc}
\hline \hline & & non dipl & CAP-BEP & Bac-Bac+2 & Sup \\
\hline Paramètres & $A_{s}$ & 2.3063 & 2.9690 & 4.5065 & 6.2296 \\
spécifiques & $\eta_{s}$ & 0.4748 & 0.5023 & 0.4915 & 0.5280 \\
& $\widetilde{b}_{s}$ & 0.9817 & 0.9759 & 0.9700 & 0.9671 \\
\hline \multirow{3}{*}{ Valeurs d'équilibre } & $p_{s} A_{s}$ & 2.9895 & 3.2120 & 3.9844 & 5.7019 \\
\cline { 2 - 3 } & $\sum_{s} \omega_{s} w_{s}$ & 0.7405 & 0.7961 & 0.9874 & 1.4137 \\
\hline \hline
\end{tabular}

TABLE 3 - Calibrations basées sur les restrictions d'état stationnaire

22. Les données sur le salaire horaire moyen par diplôme pour l'année 2014 sont données par Arnault (2018) 
Calibrations basées sur la crise de la COVID-19. On utilise la série du nombre de chômeurs données par l'OCDE à laquelle on ajoute le nombre d'EQTP effectivement placés en activité partielle. ${ }^{23}$

Les chroniques de chocs de PGF (amplitude de restriction du confinement) par type d'emploi, et les baisses de coût de reprise de l'activité salariée liées au chômage partiel sont calibrées de telle sorte à ce que le modèle reproduise les inégalités observées de chômage ${ }^{24}$ selon la catégorie socio-professionnelle pendant le premier confinement : ${ }^{25}$

$$
\Psi=\left\{\left\{U R_{t}\right\}_{t=\text { mars }}^{\text {septembre }},\left\{U R_{s, \text { avril }}\right\}_{s=1}^{S}\right\} \quad \text { avec } \operatorname{dim}(\Psi)=7+3=10
$$

Les données des taux de chômage par catégorie socio-professionnelle ne sont disponibles que pour le mois d'avril et nous en déduisons $U R_{s}$. Toujours pour ce mois d'avril, nous avons aussi les individus en chômage partiel par CSP, ${ }^{26}$ notée $P_{s}$. On suppose alors que la baisse des coûts par reprise d'emploi sera d'autant plus importante que le nombre de personnes au chômage partiel est grand. On a alors $\kappa_{s, t}$ qui devient $\left(1-\Upsilon \times P_{s}\right) \kappa_{s, t}$, où le facteur de proportionnalité $\Upsilon$ reste à déterminé, avec les variations de PGF $\left(A_{s, t}\right)$ induite par le confinement :

$$
\Phi=\left\{\left\{\left\{A_{s, t}\right\}_{s=1}^{S}\right\}_{t=\text { mars }}^{\text {avril }}, \Upsilon\right\} \text { avec } \operatorname{dim}(\Phi)=2 \times 4+1=9
$$

avec $\operatorname{dim}(\Phi)<\operatorname{dim}(\Psi)$, nécessaire à la sur-identification.

\subsection{Impact du premier confinement sur le chômage français}

Le panel (a) de la Figure 1 montre que la calibration de notre extension du modèle DMP reproduit la dynamique mensuelle du taux de chômage français depuis mars 2020 . Le panel (b) de la Figure 1 montre quant à lui que les amplitudes inégales de montés du chômage en fonction du diplôme sont également bien reproduites par le modèle : en incluant le chômage partiel, la hausse est de 32 pp pour les individus sans diplôme et autour de 10 pp pour ceux ayant au moins un Bac +2 .

Les Figures 2 et 3 montrent les évolutions des flux de travailleurs sous jacents à ces dynamique de stocks. Le Panel (a) de la Figure 2 montre que les embauches ont été nulles en avril, pour finalement repartir en mai et atteindre un pic en juin. Après deux mois de fortes reprises d'activité, en grande partie expliquée par le retour au travail des employés en chômage partiel, on observe un sur-ajustement, conduisant les embauches à converger

23. Voir DARES (2020)

24. voir Givord and Silhol (2020)

25. N'ayant pas de données par diplôme, nous approximons les non-diplômés par les ouvriers qui est la CSP regroupant le plus de non-diplômés, les individus ayant un BEP-CAP par les employés, CSP dans laquelle le pourcentage de BEP-CAP est le plus élevé, les Bac-Bac +2 par les professions intermédiaires, CSP dans laquelle le pourcentage de Bac-Bac +2 est le plus élevé, et enfin, les individus ayant un diplôme supérieur à un $\mathrm{Bac}+2$ par les cadres.

26. La même approximation est faite pour passer d'une information basée sur les CSP à une catégorisation pas diplôme. 


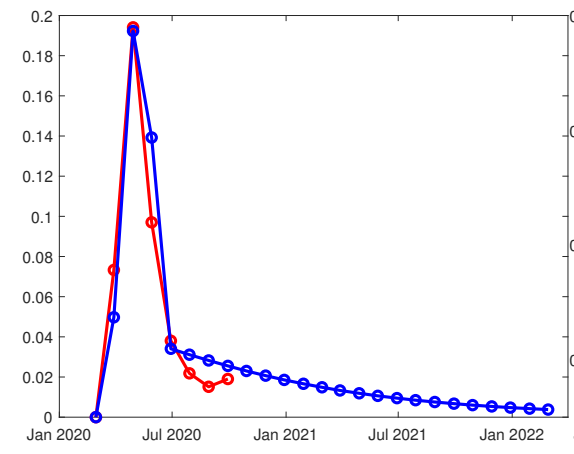

(a) Agrégé

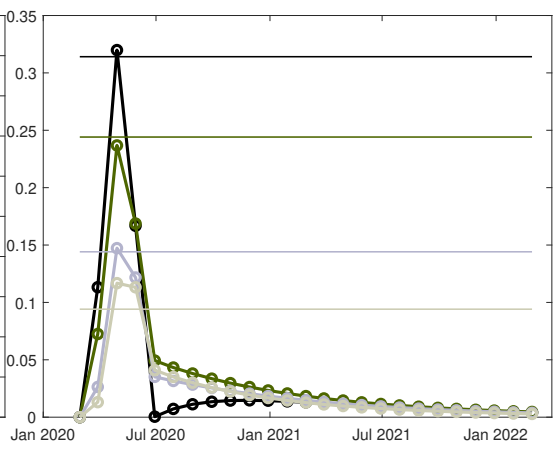

(b) Par diplôme

FIgURE 1 - Impact du premier confinement sur le taux de Chômage. Toutes les séries sont en écart à leurs valeurs d'avant crise, c'est à dire celles de février 2020. Panel (b) : les lignes en trains pleins indiquent les taux observés de chômage par diplôme en avril-mai.

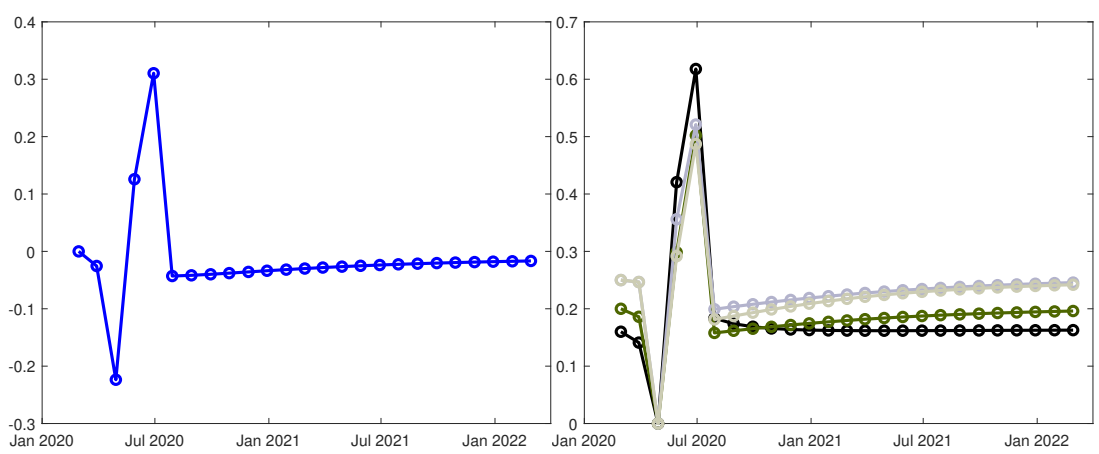

(a) Agrégé

(b) Par diplôme

FIGURE 2 - Impact du premier confinement sur le taux d'embauche. Toutes les séries sont en écart à leurs valeurs d'avant crise, c'est à dire celles de février 2020 . 
vers les niveau initial en passant légèrement en dessous de leurs niveaux de long terme. Le panel (b) de la Figure 2 indique que tous les types d'emploi sont frappés par l'arrêt des embauches en avril, et que les reprises d'emploi sont très fortes pour tous les diplômes dès mai, avec des taux de passage de l'inactivité vers l'activité entre $50 \%$ et $60 \%$, alors que ces taux sont compris entre $16 \%$ et $25 \%$ en moyenne avant la crise (voir $J F R$ dans le tableau 2). Le Panel (a) de la Figure 3 montre que le taux de séparation est de passé de 2,5\%

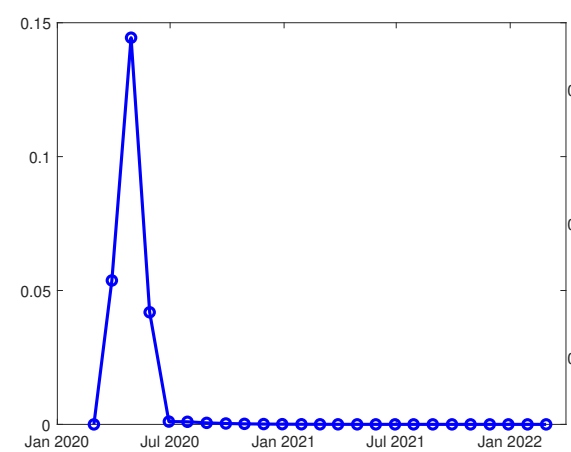

(a) Agrégé

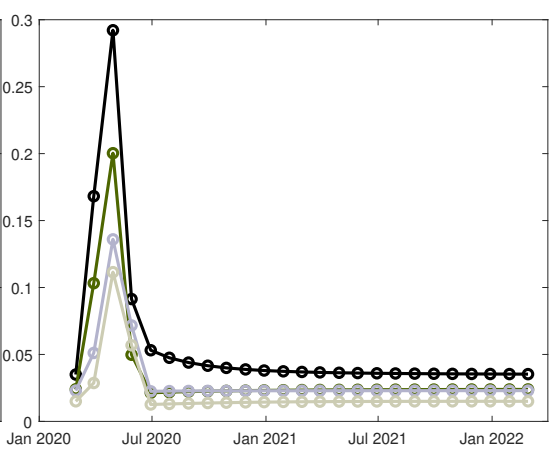

(b) Par diplôme

FIGURE 3 - Impact du premier confinement sur le taux de séparation. Toutes les séries sont en écart à leurs valeurs d'avant crise, c'est à dire celles de février 2020 .

à 17,5\% par mois entre février et avril (hausse de $15 \mathrm{pp}$ ). Comme les embauches étaient nulles en avril, ces hausses du chômage sont entièrement déterminées par les fermetures de postes de travail. Dès juin, le taux de séparation est revenu à son niveau d'avant crise. Le panel (b) de la Figure 3 montre que tous les types d'emploi ont été victimes de ces grandes vagues d'arrêt d'activité, même si elles touchent plus les moins diplômés. Comment expliquer ces ajustements si brusques et si amples? La calibration de notre

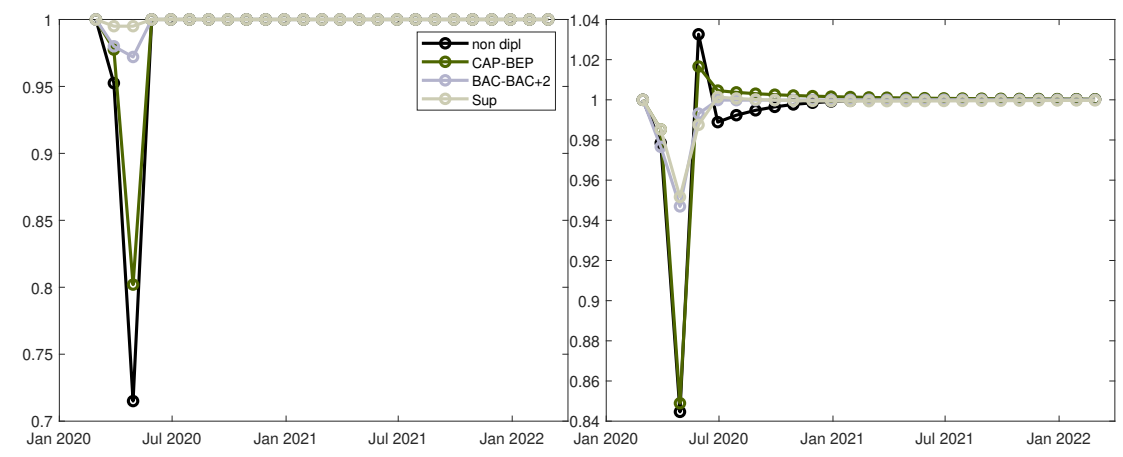

(a) PGF en volume

(b) PGF en valeur

FIGURE 4 - Impact du premier confinement sur la productivité. Toutes les séries sont en base 1 le mois d'avant crise, c'est à dire février 2020 .

modèle permet de révéler l'ampleur des "chocs COVID-19", spécifiques à chaque type d'activité. La Figure 4 représente les variations négatives de PGF, interprétées comme 
les restrictions induites par le confinement, ie. les contraintes effectives qui ont frappé de manière inattendue et différencié chaque type d'activité. La baisse quantitative de la production atteint 30\% en avril sur les postes employant des non-diplômés (panel (a) de la Figure 4), soit une perte de valeur créée par poste de 26\% (panel (b) de la Figure 4). La situation est très différente pour les postes employant des individus ayant un diplôme supérieur à $\mathrm{Bac}+2$ : la perte en volume est quasi-nulle pour une perte de valeur créée par poste de 6\% (panels (a) et (b) de la Figure 4). Les ajustements de prix relatifs viennent partiellement modérer l'impact inégalitaire des restrictions sur le échanges physique en soutenant davantage le prix relatif des biens devenant relativement plus rares.

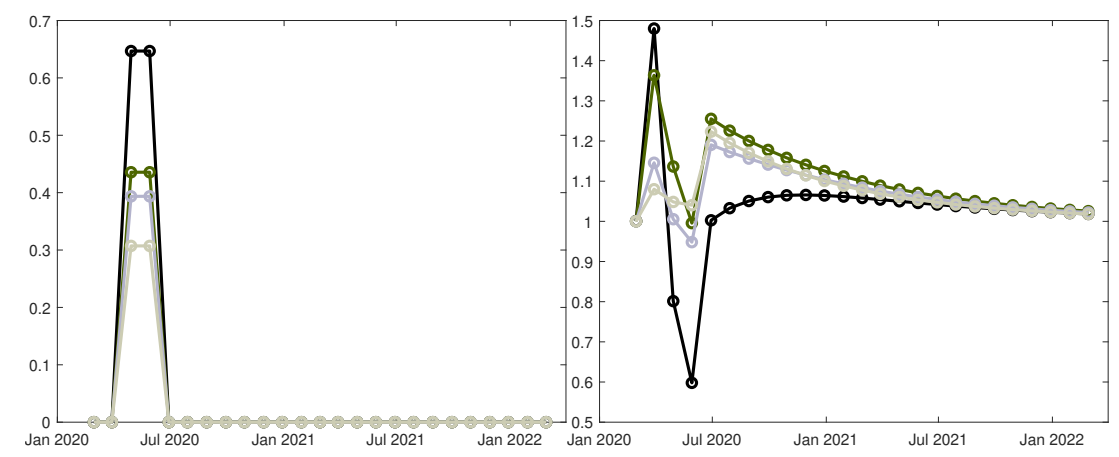

(a) Subventions

(b) Coût d'embauche

FiguRE 5 - Impact du chômage partiel sur les coûts d'embauche. Toutes les séries sont en base 1 le mois d'avant crise, c'est à dire février 2020.

La modération des coûts associés à la reprise d'activité d'une personne en chômage partiel peut également être révélée par notre modèle grâce aux données observées pendant la période de déconfinement. Cette modération transitoire des coûts s'interprète comme l'efficacité productive des mesures de chômage partiel. ${ }^{27}$ Le panel (a) de la Figure 5 donne l'amplitude de la baisse du coût en mai et juin $\left(\Upsilon \times P_{s}\right)$, alors que le panel (b) de la Figure 5 donne l'évolution induite du coût unitaire de reprise d'emploi, ie. $\left(1-\Upsilon \times P_{s}\right) \kappa_{s, t}$. Pour rendre compte de la rapidité de la résorption des niveaux de chômage observés lors du déconfinement, nous évaluons que le chômage partiel revient à baisser de $90 \%$ les coûts unitaires de reprise de l'activité d'un poste employant un non-diplômé et de $7 \%$ ceux associés à la reprise d'activité pour les personnes ayant au moins un $\mathrm{Bac}+2$.

La Figure 6 compare notre simulation de référence avec un scénario sans la baisse des coûts associés à la reprise d'emploi des individus en chômage partiel.28. Le modèle prévoit que le taux de chômage agrégé aurait été de 3 pp plus élevé en janvier 2021 qu'il ne l'était en février 2020 (soit un taux de chômage à 10,7\%), alors qu'il n'aurait été plus élevé "que" de 2 pp grâce au chômage partiel (soit un taux de chômage à 9,7\%). Au delà de ces effets à long terme, soulignant l'intérêt de toute mesure accélérant la reprise

27. Notre modèle faisant l'hypothèse de neutralité face au risque des agents économiques, il n'est possible d'évaluer le surplus de bien être associé au lissage de la consommation permis par l'assurance fournie par le chômage partiel. Nous nous focalisons donc uniquement sur les gains en emploi.

28. Ce scénario est simulé sans tenir compte de la seconde vague, discutée dans la section 3.4 


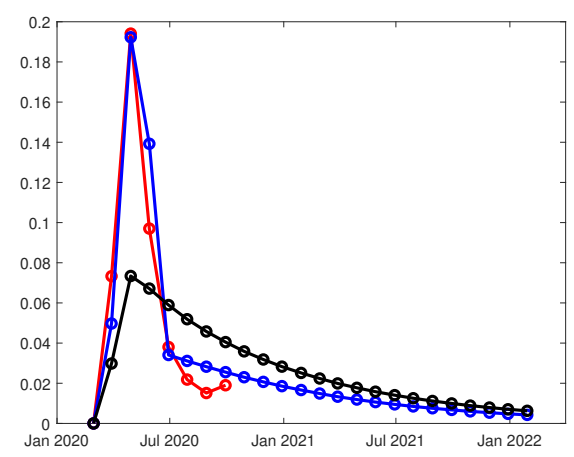

FigURE 6 - Impact du chômage partiel sur le chômage total. Rouge : données; bleue : modèle de référence; noir : modèle sans les baisses de coût d'une reprise d'activité liées au chômage partiel. Toutes les séries sont en écart à leurs valeurs d'avant crise, c'est à dire celles de février 2020 .

d'emploi sur la persistance du chômage, la simulation contre-factuelle montre aussi que le chômage partiel facilite et donc amplifie les mises à pied : comme l'employeur anticipe que la reprise d'emploi sera moins coûteuse, il aura tendance à se séparer plus facilement de ses employés, la stratégie dite de "rétention de la main d'oeuvre" étant moins valorisée. Mais ces forts ajustements à la hausse du chômage sont compensés par la très forte reprise d'activité dès le début du déconfinement, ce qui tend à faire plaider en faveur de ces mesures, même si leurs coûts lors des deux mois de confinement sont très élevés.

\subsection{Quel impact aurait eu un confinement plus souple mais plus long?}

Le premier confinement a été très strict, mais assez court : peu de déplacements étaient autorisés, les établissements scolaires fermés et donc les activités salariés fortement contraintes. Quelles auraient été l'impact d'une stratégie de confinement moins strict, mais plus longue? Afin d'isoler l'impact de la durée du confinement, on calibre le scénario contre-factuel de telle sorte que la somme actualisée des diminutions de PGF soit identique à celle du scénario de référence. Le panel (b) de la Figure 7 permet de comparer les variations de PGF entre un confinement strict de 1,5 mois et un confinement souple de 3 mois : ce scénario contre-factuel implique une baisse de la PGF de 11\% (0,33\%) pour les non-qualifiés (les diplômés de l'enseignement supérieur), alors qu'elle était de 30\% $(0,5 \%)$ dans le scénario de référence (voir panel (a) de la Figure 7). Pour la baisse du coût unitaire d'une reprise d'activité lié au chômage partiel, on suppose qu'elle est identique à celle du premier confinement (voir panel (a) de la Figure 5). Le Panel (c) de la Figure 8 montre qu'un confinement plus doux permet d'éviter plus d'un tiers de l'énorme pic des séparations d'avril. Évidement, avec un confinement plus long, des séparations plus importantes que leur niveau de long terme s'observent encore en juin, alors que ce n'était pas le cas dans le scénario de référence. Toutefois, ces excès de licenciement en mai et juin pour le scénario contre-factuel restent modérés. Le Panel (b) montre que la dynamique des reprises d'emploi est similaire dans les deux expériences, avec un retard d'un mois pour un confinement plus long. Finalement, le Panel (a) de la Figure 8 montre qu'une 


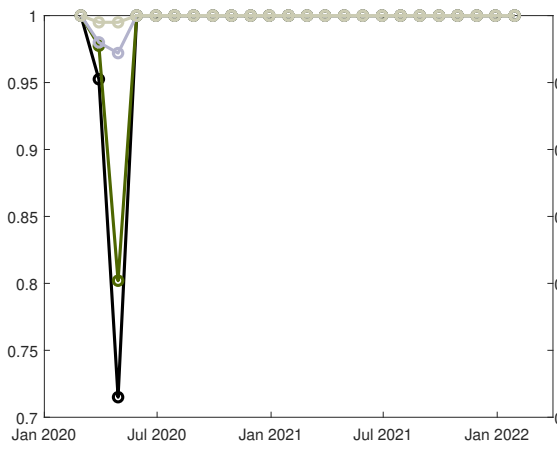

(a) Confinement court et strict

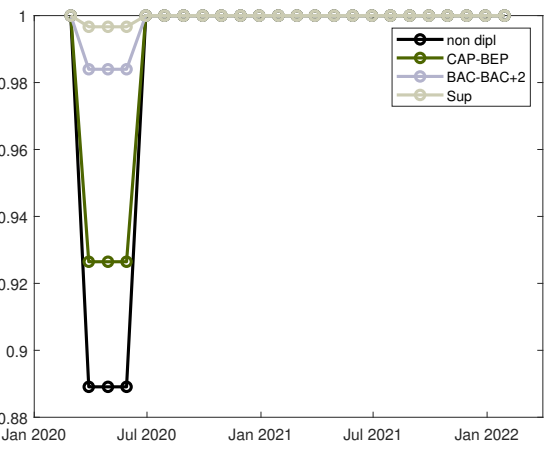

(b) Confinement long et souple

FIgURE 7 - Comparaison des variations de PGF : confinement strict mais cours versus souple mais long. Toutes les séries sont en base 1 le mois d'avant crise, c'est à dire février 2020 .

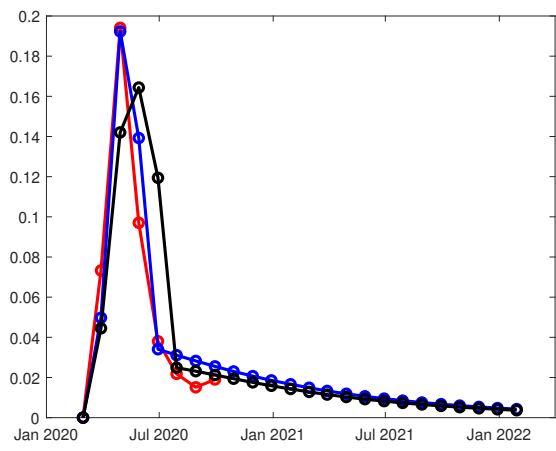

(a) Taux de chômage

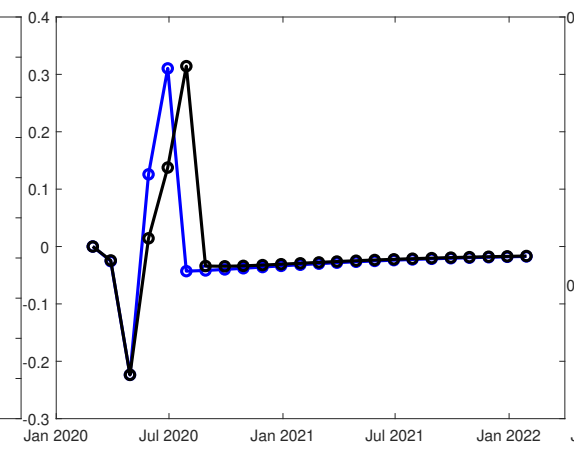

(b) Taux d'embauche

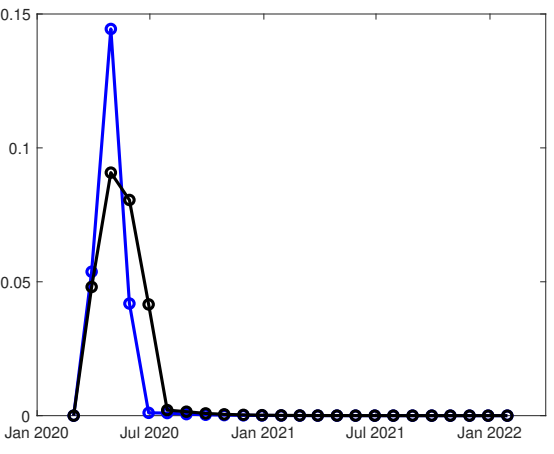

(c) Taux de séparation

Figure 8 - Comparaison entre un confinement brusque mais cours et un confinement souple mais long. Rouge : données; bleue : modèle de référence; noir : modèle avec un confinement plus souple mais plus long. Toutes les séries sont en écart à leurs valeurs d'avant crise, c'est à dire celles de février 2020 . 
stratégie de confinement moins stricte mais plus longue permet de revenir plus vite au niveau de chômage d'avant crise. En effet, grâce à son effet plus modéré sur les séparations, un confinement moins stricte permettant de laisser ouvert davantage de postes.

\subsection{Quel sera l'impact du second confinement?}

Dans cette dernière section, nous présentons deux simulations pour le second confinement, commençant tous les deux à la mi-octobre (début du couvre-feu) : l'une évaluant l'impact d'un second confinement plus long et moins strict que celui débuté en mars, et l'autre identique à celui de mars. La Figure 9 montre que la stratégie d'un confinement plus souple permet de contenir la hausse du chômage et permet de limiter les mises à pied, ce qui implique un retour du chômage à son niveau d'avant-crise légèrement plus rapide que dans le cas d'un confinement plus strict.

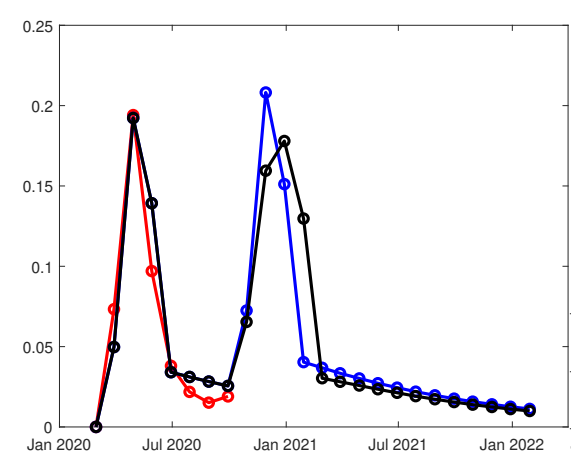

(a) Chômage

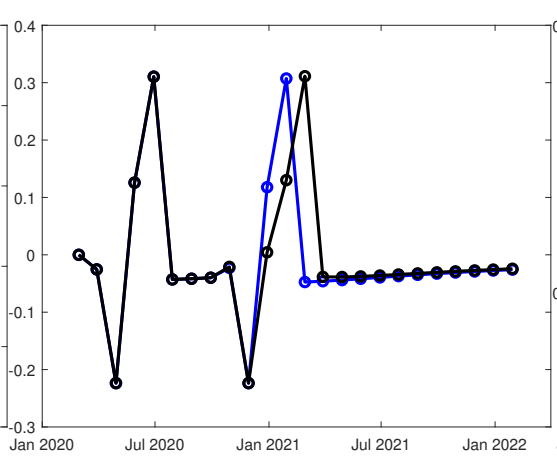

(b) Taux d'embauche

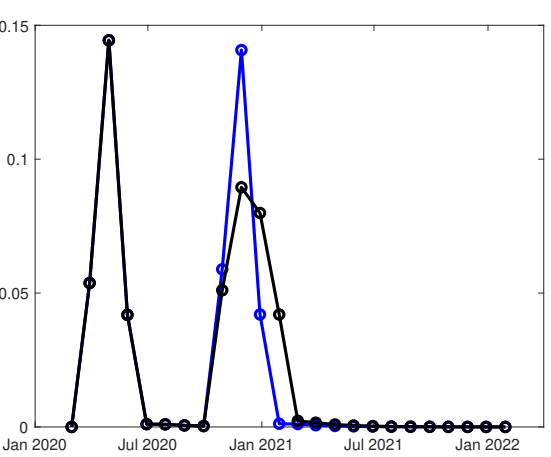

(c) Taux de séparation

Figure 9 - Une deuxième vague en Octobre. Toutes les séries sont en écart à leurs valeurs d'avant crise, c'est à dire celles de février 2020.

\section{Conclusion}

Les restrictions induites par le confinement sont assez difficiles à modéliser dans les modèle macro-économiques. Dans cet article nous proposons une piste en enrichissant le modèle DMP pour analyser l'impact de la crise COVID-19 sur le marché du travail français. Notre approche permet de révéler (i) l'amplitude des restrictions sur la production induites par le confinement, et (ii) la baisse des coûts des reprises d'activité induite par les mesures de chômage partiel qui sont compatibles avec l'ampleur observée du pic du chômage d'avril ainsi que sa très rapide résorption. Elle permet également d'identifier les différences par niveau de diplôme induite par le confinement. Nous montrons également qu'un confinement souple et plus long est préférable à un confinement strict et court de par son effet plus mesuré sur les séparations. Ainsi, le second confinement, plus souple que le premier, semble être plus favorable aux ajustements du marché du travail. 


\section{Références}

Adams-Prassl, A., Boneva, T., Golin, M. and Rauh, C. (2020), 'Inequality in the Impact of the Coronavirus Shock : Evidence from Real Time Surveys', Journal of Public Economics 189.

Adjemian, S., Karame, F. and Langot, F. (2019), On nonlinearities in the unemployment dynamic, mimeo, CEPREMAP.

Arnault, S. (2018), Salaire horaire : l'importance de la catégorie socioprofessionnelle et du diplôme, Insee Focus 116.

Barrero, J., Bloom, N. and Davis, J. (2020), COVID-19 Is Also A Reallocation Shock, NBER Working Paper Series 27137.

Bartik, W., Bertrand, M., Lin, F., Rothstein, J. and Unrath, M. (2020), Measuring the labor market at the onset of the covid-19 crisis, NBER Working Paper Series 27613.

Beck, S. and Vidalenc, J. (2017), Une photographie du marché du travail en 2016, InseePremière 1648.

Bernstein, J., Richter, A. and Throckmorton, A. (2020), COVID-19 : A View from the Labor Market, Federal Reserve Bank of Dallas Working Paper 2010.

Birinci, S., Karahan, F., Mercan, Y. and See, K. (2020), Labor market policies during an epidemic, Federal Reserve Bank of New York Staff Reports 943.

Blanchard, J. O. and Diamond, P. (1994), 'Ranking, unemployment duration, and wages', The Review of Economic Studies 61, 417-434.

Blanchard, O. and Gali, J. (2010), 'Labor markets and monetary policy : A new keynesian model with unemployment', American Economic Journal : Macroeconomics 2, 1-30.

Bloom, N. (2009), 'The Impact of Uncertainty Shocks', Econometrica 77, 623-685.

Bloom, N., Floetotto, M., Jaimovich, N., Saporta, E. and Terry, J. (2018), 'Really Uncertain Business Cycles', Econometrica 86, 1031-1065.

Christiano, L., Eichenbaum, M. and Trabandt, M. (2016), 'Unemployment and business cycles', Econometrica 84(4), 1523-1569.

Cortes, G. and Forsythe, E. (2020), Impacts of the COVID-19 Pandemic and the CARES Act on Earnings and Inequality, IZA 13643.

Daly, M., Hobijn, B. and Lucking, B. (2012), Why has wage growth stayed strong?, Federal Reserve Bank of San Francisco Economic Letter. Issue Apr2.

DARES (2020), Situation sur le marché du travail durant la crise sanitaire, Dares tableau de bord. 
Den Haan, W., Ramey, G. and Watson, J. (2000), 'Job destruction and propagation of shocks', American Economic Review 90, 482-498.

Diamond, P. (1982), 'Wage determination and efficiency in search equilibrium', Review of Economic Studies 49, 217-227.

Elsby, M. and Solon, G. (2019), 'How prevalent is downward rigidity in nominal wages? international evidence from payroll records and pay slips', The Journal of Economic Perspectives 33(3), 185-201.

Engbom, N. (2019), Application cycles, Society for Economic Dynamics 1170.

Fana, M., Tolan, S., Torrejón, S., Brancati, U. and Fernández-Macías, E. (2020), The covid confinement measures and eu labour markets, JRC 120578.

Ferraro, D. (2018), 'The Asymmetric Cyclical Behavior of the U.S. Labor Market', Review of Economic Dynamics 30, 145-162.

Ferraro, D. (2020), 'Fast Rises, Slow Declines : Asymmetric Unemployment Dynamics with Matching Frictions', Journal of Money, Credit and Banking Forthcoming.

Fontaine, I., Gálvez-Iniesta, I., Gomes, P. and Vila-Martind, D. (2020), 'Labour market flows : Accounting for the public sector', Labour Economics 62(101770).

Gallant, J., Kroft, K., Lange, F. and Notowidigdo, M. (2020), Temporary Unemployment And Labor Market Dynamics During the COVID-19 Recession, NBER Working Paper series 27924.

Givord, P. and Silhol, J. (2020), Confinement : des conséquences économiques inégales selon les ménages, Insee-Première 1822.

Gregory, V., Menzio, G. and Wicze, G. D. (2020), Pamndemic recession : L or V-shaped?, NBER Working Paper Series 27105.

Guggemos, F. and Vidalenc, J. (2014), Une photographie du marché du travail en 2013, Insee-Première 1516.

Hall, R. E. (2005), 'Employment fluctuations with equilibrium wage stickiness', American Economic Review 95(1), 50-65.

Hall, R. E. and Milgrom, P. R. (2008), 'The limited influence of unemployment on the wage bargain', American Economic Review 98(4), 1653-1674.

Hall, R. and Kudlyak, M. (2020), Why Has the US Economy Recovered So Consistently from Every Recession in the Past 70 Years?, NBER Working Paper series 27234.

Jardim, E., Solon, G. and Vigdor, J. (2019), How Prevalent Is Downward Rigidity in Nominal Wages? Evidence from Payroll Records in Washington State, NBER Working Paper series 25393. 
Jauneau, Y. and Vidalenc, J. (2020), Durée travaillée et travail à domicile pendant le confinement : des différences marquées selon les professions, Insee Focus 207.

Kandoussi, M. and Langot, F. (2020), On the heterogeneous impacts of the COVID-19 lockdown on US unemployment, Working paper.

Kapicka, M. and Rupert, P. (2020), Labor markets during pandemics, Mimeo. UC Santa Barbara.

Krause, M. and Lubik, T. A. (2007), 'The (ir)relevance of real wage rigidity in the new keynesian model with search frictions', Journal of Monetary Economics 54(3), 706-727.

Kurmann, A. and McEntarfer, E. (2019), Downward Nominal Wage Rigidity in the United States : New Evidence from Worker-Firm Linked Data, Center for Economic Studies (Census) 19-07.

Leduc, S. and Liu, Z. (2019), 'The Weak Job Recovery in a Macro Model of Search and Recruiting Intensity', 12(1), 310-343.

Lise, J. and Robin, J.-M. (2017), 'The macro-dynamics of sorting between workers and firms', American Economic Review 107(4), 1104-1135.

Molavi, P. (2018), A theory of dynamic selection in the labor market, Technical report, Department of Economics, MIT.

Mortensen, D. (1982), The matching process as a noncooperative bargaining game, in J. J. McCall, ed., 'The Economics of Information and Uncertainty', University of Chicago Press.

Nicolas Petrosky, N. and Zhang, L. (2020), 'Unemployment crises', Journal of Monetary Economics forthcoming.

Pissarides, C. (1985), 'Short-run dynamics of unemployment, vacancies, and real wages', American Economic Review 75, 676-690.

Robin, J.-M. (2011), 'On the dynamics of unemployment and wage distributions', Econometrica 79(5), 1327-1355. 


\section{A Le problème de l'entreprise}

La fonction de production est alors donnée par

$$
Y_{s, t}=A_{s, t} N_{s, t} \frac{1}{1-G_{s, t}\left(\alpha_{t}^{r}\right)} \int_{\alpha_{s, t}^{r}}^{+\infty} \alpha d G_{s, t}(\alpha)=A_{s, t} \widetilde{\alpha}_{s, t} N_{s, t}
$$

où on note $\widetilde{\alpha}_{s, t}=\frac{\int_{\alpha_{s, t}^{r}}^{+\infty} \alpha d G_{s, t}(\alpha)}{1-G_{s, t}\left(\alpha_{s, t}^{r}\right)}$ et $A_{s, t}$ la productivité spécifique à chaque niveau de diplôme.

On notant $\widetilde{w}_{s, t}=\frac{\int_{\alpha_{s, t}^{r}}^{+\infty} w_{s, t}(\alpha) d G_{s, t}(\alpha)}{1-G_{s, t}\left(\alpha_{s, t}^{r}\right)}$, le programme de l'entreprise est :

$$
\begin{aligned}
\mathcal{V}_{s, t}\left(N_{s, t}, A_{s, t}\right)= & \max _{V_{s, t}, N_{s, t}, \alpha_{s, t}^{r}} D_{s, t}+\beta_{t} \mathcal{V}_{s, t+1}\left(N_{s, t+1}, A_{t+1}\right) \\
\text { s.t } & \left\{\begin{aligned}
D_{s, t} & =N_{s, t}\left(p_{s, t} A_{s, t} \widetilde{\alpha}_{s, t}-\widetilde{w}_{s, t}\right)-p_{s, t} \kappa_{s, t} V_{s, t} \\
N_{s, t+1} & =\left(1-s_{s, t+1}\right)\left(N_{s, t}+q\left(\theta_{s, t}\right) V_{s, t}\right) \\
V_{s, t} & \geq 0
\end{aligned}\right.
\end{aligned}
$$

Les conditions de première ordre $(\mathrm{CPO})$ sont :

$$
\begin{aligned}
0= & -p_{s, t} \kappa_{s, t}+q\left(\theta_{s, t}\right) \beta_{t}\left(1-s_{s, t+1}\right) \frac{\partial \mathcal{V}_{s, t+1}}{\partial N_{s, t+1}}+\lambda_{s, t} q\left(\theta_{s, t}\right) \\
0= & \frac{\partial N_{s, t}}{\partial \alpha_{s, t}^{r}}\left(p_{s, t} A_{s, t} \widetilde{\alpha}_{s, t}-\widetilde{w}_{s, t}\right)+N_{s, t}\left(p_{s, t} A_{s, t} \frac{\partial \widetilde{\alpha}_{s, t}}{\partial \alpha_{s, t}^{r}}-\frac{\partial \widetilde{w}_{s, t}}{\partial \alpha_{s, t}^{r}}\right) \\
& +\beta_{t} \frac{\partial \mathcal{V}_{s, t+1}}{\partial N_{s, t+1}} \frac{\partial N_{s, t+1}}{\partial N_{s, t}} \frac{\partial N_{s, t}}{\partial \alpha_{s, t}^{r}} \\
\frac{\partial \mathcal{V}_{s, t}}{\partial N_{s, t}}= & p_{s, t} A_{s, t} \widetilde{\alpha}_{s, t}-\widetilde{w}_{s, t}+\beta_{t}\left(1-s_{s, t+1}\right) \frac{\partial \mathcal{V}_{s, t+1}}{\partial N_{s, t+1}}
\end{aligned}
$$

Les conditions de Kuhn-Tucker sont données par

$$
q_{s}\left(\theta_{s, t}\right) V_{s, t} \geq 0, \quad \lambda_{s, t} \geq 0, \quad \text { and } \quad \lambda_{s, t} q_{s}\left(\theta_{s, t}\right) V_{s, t}=0 .
$$

Sachant que $1-s_{s, t}=\left(1-s_{s}\right)\left(1-G_{s}\left(\alpha_{s, t}^{r}\right)\right)$ et en utilisant

$$
\begin{aligned}
\frac{\partial N_{s, t}}{\partial \alpha_{s, t}^{r}} & =-\left(1-s_{s}\right)\left(N_{s, t-1}+q\left(\theta_{s, t-1}\right) V_{s, t-1}\right) d G_{s}\left(\alpha_{s, t}^{r}\right)=-\left(1-s_{s}\right) \frac{N_{s, t}}{1-s_{s, t}} d G_{s}\left(\alpha_{s, t}^{r}\right) \\
\frac{\partial \widetilde{\alpha}_{s, t}}{\partial \alpha_{s, t}^{r}} & =\frac{d G_{s}\left(\alpha_{s, t}^{r}\right)}{1-G_{s}\left(\alpha_{s, t}^{r}\right)}\left(\widetilde{\alpha}_{s, t}-\alpha_{s, t}^{r}\right) \\
\frac{\partial \widetilde{w}_{s, t}}{\partial \alpha_{s, t}^{r}} & =\frac{d G_{s}\left(\alpha_{s, t}^{r}\right)}{1-G_{s}\left(\alpha_{s, t}^{r}\right)}\left(\widetilde{w}_{s, t}-w_{s, t}\left(\alpha_{s, t}^{r}\right)\right),
\end{aligned}
$$

l'équation (6) peut être réécrite comme suit :

$$
0=p_{s, t} A_{s, t} \alpha_{s, t}^{r}-w_{s, t}\left(\alpha_{s, t}^{r}\right)+\left(\frac{p_{s, t} \kappa_{s, t}}{q\left(\theta_{s, t}\right)}-\lambda_{s, t}\right)
$$


Les CPO conduisent aux conditions inter-temporelles de destruction et de création suivantes :

$$
\begin{aligned}
J_{s, t}\left(\alpha_{s, t}\right) & =p_{s, t} A_{s, t} \alpha_{s, t}-w\left(\alpha_{s, t}\right)+\beta_{t}\left(1-s_{s, t+1}\right) J_{s, t+1} \quad \forall \alpha_{s, t} \geq \alpha_{s, t}^{r} \\
J_{s, t}\left(\alpha_{s, t}^{r}\right) & =0 \\
J_{s, t} & =p_{s, t} A_{s, t} \widetilde{\alpha}_{s, t}-\widetilde{w}_{s, t}+\beta_{t}\left(1-s_{s, t+1}\right) J_{s, t+1} \\
\frac{p_{s, t} \kappa_{s, t}}{q\left(\theta_{s, t}\right)}-\lambda_{s, t} & =\beta_{t}\left(1-s_{s, t+1}\right) J_{s, t+1}
\end{aligned}
$$

où $J_{s, t}=\frac{\partial \mathcal{V}_{s, t}}{\partial N_{s, t}}$ représente la valeur marginale de l'emploi, définie par $J_{s, t} \equiv \frac{\int_{\alpha_{s, t}^{r}}^{+\infty} J_{s, t}(\alpha) d G_{s, t}(\alpha)}{1-G_{s, t}\left(\alpha_{s, t}^{r}\right)}$, avec $J_{s, t}(\alpha)$ la valeur marginal d'un emploi après la réalisation du choc idiosyncratique de productivité $\alpha$. Comme $J_{s, t}(\alpha) \geq 0, \forall \alpha \geq \alpha_{s, t}^{r}$, la valeur moyenne de l'emploi, définit par $J_{s, t}$, est nécessairement positive. La condition intertemporelle de destruction d'emplois indique que les pertes actuelles $\left(p_{s, t} A_{s, t} \alpha_{s, t}^{r}-w_{s, t}\left(\alpha_{s, t}^{r}\right)\right)$ doivent être compensées par les gains futurs attendus générés par l'emploi. Les embauches sont basées sur l'espérance de la valeur moyenne d'un poste de travail $J_{s, t+1}$, car $\alpha$ est observé après l'appariement.

En utilisant l'équation du salaire et $\alpha \in[0,+\infty)$ quand la distribution suit une loi de distribution log-normal, le seuil de productivité d'équilibre est :

$$
\alpha_{s, t}^{r}=\max \left\{0 ; \frac{1}{\left(1-\eta_{s}\right) p_{s, t} A_{s, t}}\left[\left(1-\eta_{s}\right) b_{s}+\eta p_{s, t} \kappa_{s, t} \theta_{s, t}-\left(\frac{p_{s, t} \kappa_{s, t}}{q\left(\theta_{s, t}\right)}-\lambda_{s, t}\right)\right]\right\} .
$$

Les CPO du programme de l'entreprise conduisent à la condition de création d'emplois intertemporelle suivante :

$$
\frac{p_{s, t} \kappa_{s, t}}{q_{s}\left(\theta_{s, t}\right)}-\lambda_{s, t}=\beta_{t}\left[\left(1-s_{s, t+1}\right)\left(p_{s, t+1} A_{s, t+1} \widetilde{\alpha}_{s, t+1}-\widetilde{w}_{s, t+1}+\frac{p_{s, t+1} \kappa_{s, t+1}}{q_{s}\left(\theta_{s, t+1}\right)}-\lambda_{s, t+1}\right)\right]
$$

Quand $\lambda_{s, t}=0$, l'équilibre est le même que dans le modèle DMP. Cependant, quand $\lambda_{t}>$ 0 , on a $V_{s, t}=0$, et la solution est contrainte à $\theta_{s, t}=0$ and $N_{t}=\left(1-s_{s}\right)\left(1-G_{s}\left(\alpha_{s, t}^{r}\right)\right) N_{s, t-1}$. 


\section{B Graphiques : comparaison entre deux types de confi- nement}

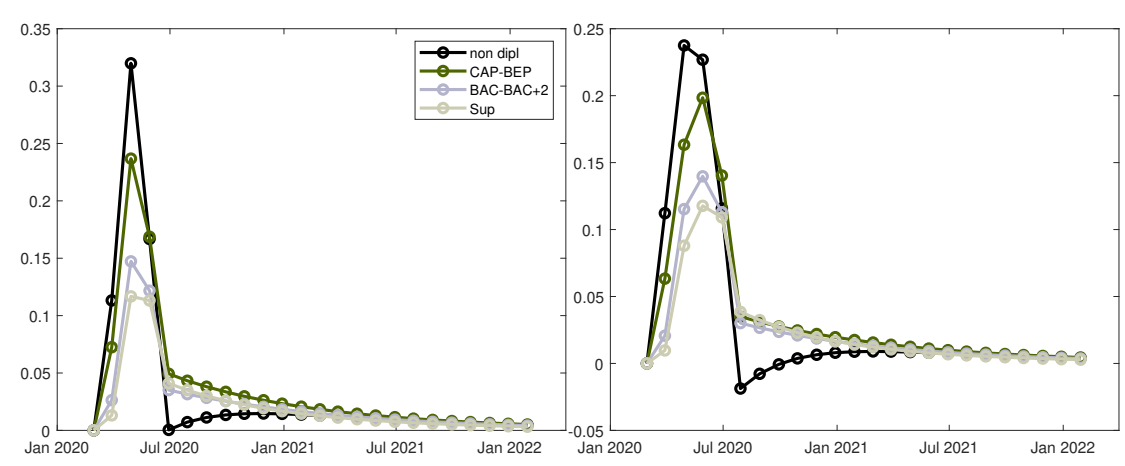

(a) Confinement court et strict $\quad$ (b) Confinement long et souple

Figure 10 - Chômage

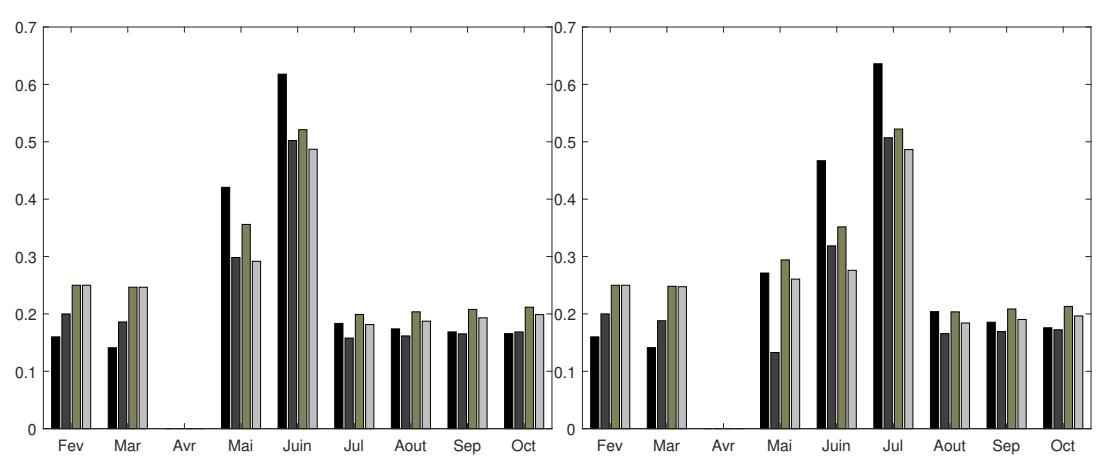

(a) Confinement court et strict (b) Confinement long et souple

Figure 11 - Taux d'embauche. 


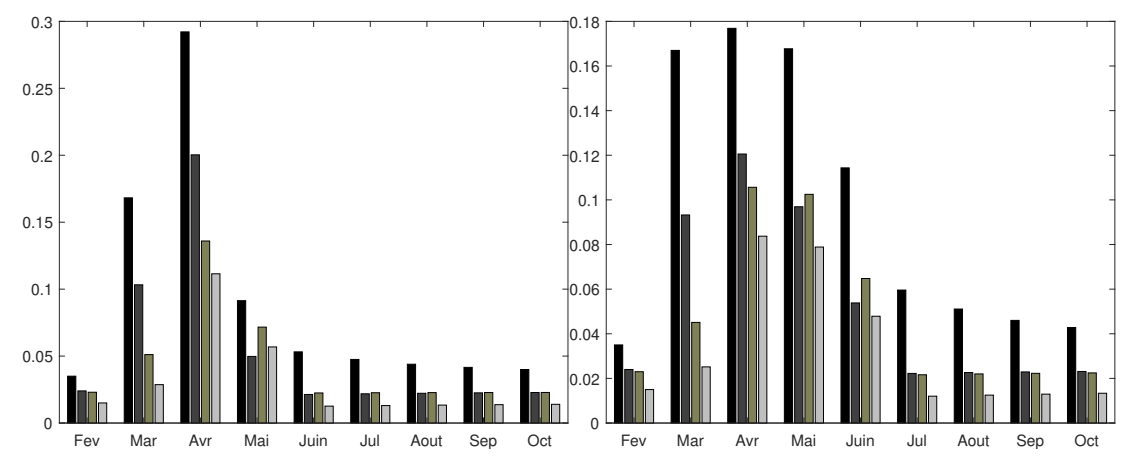

(a) Confinement court et strict $\quad$ (b) Confinement long et souple

Figure 12 - Taux de séparation.

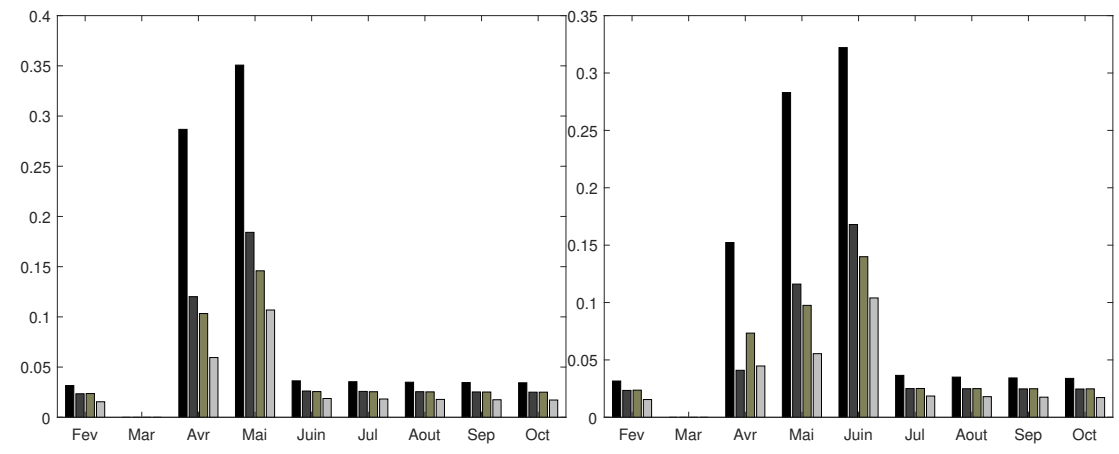

(a) Confinement court et strict $\quad$ (b) Confinement long et souple

FigURE 13 - Nombre de postes vacants. 